\title{
Phylogenetic analysis of genes encoding the enzymes of plant amino acids catabolism in representatives of the genus Methylobacterium
}

\author{
M. Krohaleva ${ }^{*}$, D. Fedorov², G. Ekimova² \\ ${ }^{1}$ Ural Federal University named after the first President of Russia B.N.Yeltsin, Ekaterinburg, Russia \\ ${ }^{2}$ Institute of Biochemistry and Physiology of Microorganisms RAS, Pushchino, Russia \\ *e-mail:krohaleva-mariya@mail.ru
}

Key words: Methylobacterium, phytosymbiosis, ACC-deaminase, D-cysteine desulfhydrase

Motivation and Aim: Aerobic methylobacteria use oxidized and substituted methane derivatives as the sources of carbon and energy. Methylobacteria can stimulate growth and development of plants due to biosynthesis of phytohormones and vitamins. One of the mechanisms of the beneficial effects of bacteria is the reduction of level of the "stress" hormone ethylene in plant tissues due to the activity of 1-aminocyclopropane1-carboxylate (ACC) deaminase [1]. An effective approach of assessing the ability of bacteria to phytosymbiosis is the search of genes whose products can stimulate the growth of plants, using PCR with degenerate primers. In the case of the ACC deaminase gene $(a c d S)$, such analysis is complicated by the presence in the genomes of some bacteria of a gene encoding D-cysteine desulfhydrase $(d c y D)$, an enzyme whose amino acid sequence exhibits a high level of identity with the sequences of ACC deaminases. The activity of this enzyme probably increases the resistance of plants to phytopathogens due to the formation of hydrogen sulphide, which functions as a fungicide [2].

Methods and Algorithms: 48 species of the genus Methylobacterium were studied. The following methods were used: DNA extraction, PCR, cloning and sequencing of DNA fragments. For amino acid and nucleotide sequences phylogenetic analysis neighbor joining/UPGMA methods were used realized in the MEGA6 [3].

Results: Taking together with genomic data, either $a c d S$ or $d c y D$ were found and/or sequenced in 29 of the 48 type strains of different species of the genus Methylobacterium. Of these, 18 strains possess the $a c d S$ gene, whereas 13 strains - the $d c y D$ gene, two species have two genes simultaneously and, therefore, can stimulate the growth and development of plants due to the activity of both enzymes.

Conclusion: For the first time it is shown that the distribution of the genes for acdS and $d c y D$ Methylobacterium species is subject to certain phylogenetic regularities, since two groups of species containing the gene $a c d S$ and one gene of $d c y D$ have been found.

Acknowledgements: The work was supported by the grant of the Russian Fund for Basic Research RFBR (16-04-00381_a).

\section{References}

1. Glick B.R. et al. (2007) Promotion of plant growth by ACC deaminase-producing soil bacteria. European Journal Plant Pathology. 119(3):329-339.

2. Papenbrock J. et al. (2007) Characterization of cysteine-degrading and $\mathrm{H}_{2} \mathrm{~S}$-releasing enzymes of higher plants - from the field to the test tube and back. Plant Biology. 9(5):582-588.

3. Tamura K. et al. (2013) MEGA6: molecular evolutionary genetics analysis version 6.0. Molecular Biology Evolution. 30(12):2725-2729. 